\title{
Breakfast-Based Dietary Patterns and Obesity in Tehranian Adults
}

\author{
Zahra Akbarzade', Saba Mohammadpour', Kurosh Djafarian², Cain C. T. Clark³, Parivash Ghorbaninejad', Maryam Mohtashami', \\ Sakineh Shab-Bidar, ${ }^{1, *}$ \\ Departments of ${ }^{1}$ Community Nutrition and ${ }^{2}$ Clinical Nutrition, School of Nutritional Sciences and Dietetics, Tehran University of Medical Sciences, Tehran, Iran; \\ ${ }^{3}$ Centre for Sport, Exercise and Life Sciences, Coventry University, Coventry, UK
}

Background: Breakfast is an important meal of the day that contributes to an overall healthy dietary pattern, better nutrient intake, and diet quality. This study sought to investigate the relationship between breakfast patterns and general and central obesity among middle-aged adults.

Methods: In this cross-sectional study of 840 apparently healthy women and men, aged 20-59 years, we assessed usual dietary intake by means of three 24-hour dietary recalls and we took anthropometric measurements. Dietary patterns were subsequently identified by factor analysis. To assess the association between breakfast composition and central and general obesity, logistic regression analysis was performed.

Results: We identified three major dietary patterns by factor analysis: the "bread and grains, meat products, and coffee" dietary pattern, the "sweets, tea and coffee" dietary pattern, and the "fruits, vegetables, and eggs" dietary pattern. Those people in the third tertile of the "sweets, tea and coffee" dietary category had a greater chance of having central obesity (odds ratio, 1.80; 95\% confidence interval, 1.25-2.59; $P=0.001$ ). Moreover, higher adherence to the "bread and grains, meat products, and coffee" pattern increased the chance of central obesity (odds ratio, $1.67 ; 95 \%$ confidence interval, $1.47-1.97 ; P=0.03$ ).

Conclusion: Overall, our results suggest that specific breakfast dietary patterns are associated with increased odds of central obesity in Iranian adults.

Key words: Dietary patterns, Breakfast, Adults, Cross-sectional study, General obesity, Central obesity
Received April 29, 2020

Reviewed June 23, 2020

Accepted July 20, 2020

${ }^{*}$ Corresponding author

Sakineh Shab-Bidar

https://orcid.org/0000-0002-0167-7174

Department of Community Nutrition, School of Nutritional Sciences and Dietetics, Tehran University of Medical Sciences, No 44, Hojjat-dost Alley, Naderi St., Keshavarz Blvd, Tehran, Iran Tel: +98-91-11376516

Fax: +98-21-88974462

E-mail:s_shabbidar@tums.ac.ir

\section{INTRODUCTION}

Obesity is a serious community health problem worldwide and is the most prevalent metabolic disorder in both developed and developing countries. ${ }^{1}$ Moreover, obesity and overweight are prevalent in more than fifty percent of Iranian adults. ${ }^{2}$ Type of obesity and fat distribution are important factors that are inextricably linked to the progression of chronic diseases. ${ }^{3}$ General obesity, assessed by body mass index (BMI), is a major risk factor for several chronic diseases, including diabetes, cardiovascular disease, cancer, and a variety of disabling and life-threatening chronic conditions. ${ }^{4}$
Although general obesity is a good predictor of life threatening diseases, ${ }^{4}$ central obesity is also considered an independent predictor of cardio-metabolic diseases, cardiovascular risk factors, certain cancers, and morbidity and mortality.,

Both environmental and genetic factors are responsible for the high prevalence of obesity. Among environmental factors, low levels of physical activity, excess sedentary behavior, and increased energy intake are key contributors to the obesity epidemic. Because of the complexity of the human diet and interactions between foods and nutrients, assessing overall diet and its relationship with chronic disease is important. Dietary patterns consider dietary

Copyright (C) 2020 Korean Society for the Study of Obesity

(a) This is an Open Access article distributed under the terms of the Creative Commons Attribution Non-Commercial License (https://creativecommons.org/licenses/by-nc/4.o/) which permits unrestricted non-commercial use, distribution, and reproduction in any medium, provided the original work is properly cited 
complexity and the long-term effects of diet on the risk of disease development, so can better predict diet-disease associations, as compared to the assessment of single nutrients. ${ }^{7}$ Moreover, foods are consumed as meals and snacks, and thus investigation of diet quality across meals is a pragmatic approach to help elucidate important diet-disease relationships.

Breakfast as the first meal of the day contributes to an overall healthy dietary pattern, better nutrient intake, and diet quality. ${ }^{8}$ The effects of breakfast on obesity are controversial. There is an abundance of evidence to suggest that breakfast consumption is associated with overweight and obesity. ${ }^{9,10}$ However, Chatelan et al. ${ }^{9}$ reported that participants who ate fruit, natural cereal flakes, nuts/ seeds, and yogurt for breakfast had a lower risk of abdominal obesity because of good dietary choices the rest of the day. In two studies involving Iranian and African-American students, respectively, skipping breakfast was associated with overweight and obesity; ${ }^{11,12}$ moreover, some studies have shown that in breakfast skippers, free fatty acids increase throughout the morning hours, resulting in decreased insulin sensitivity later in the day. ${ }^{13,14} \mathrm{~A}$ recent meta-analysis suggested that consumption of breakfast is not an effective weight loss strategy, ${ }^{15}$ whilst in another study of Korean adults, a Western breakfast pattern that included eggs, Western grains, and processed meat was associated with an increased risk of metabolic syndrome, of which abdominal obesity is a key component, compared to a fruit, nuts, and vegetables breakfast pattern. ${ }^{16}$

In previous studies, dietary patterns have been identified based on average daily intake, because food is consumed at different times in different meals. In the other words, the focus of nutritional epidemiology has been on individual nutrients with a more recent shift in focus to dietary patterns. However, little data exist on the quality of diet and dietary intake at the level of a "meal." Identifying meal-based dietary patterns may help the public to achieve the recommended daily intake of foods and nutrients and adhere to dietary guidelines, and may also enable the identification of diet-disease associations. Previous literature that has investigated meal patterns has predominantly been conducted in Western countries. Given the dearth of studies originating from Middle Eastern countries, we sought to investigate the relationship between breakfast patterns and general and central obesity in an Iranian population.

\section{METHODS}

\section{Study population and design}

This study was a cross-sectional, population-based survey of 850 adults aged 20-59 years living in Tehran. Ten participants were excluded for various reasons, resulting in a total of 840 participants in the final analysis. Sampling was done by dividing the health centers of Tehran into five regions: north, south, east, west, and center; then randomly recruiting a proportional amount of participants from the five regions. Study criteria included an age between 20 and 59 years, desire to participate in the survey, self-certified to be healthy, residents of Tehran, and members of a health center. Sample collection was facilitated by coordination between the Health Bureau of the Municipality of Tehran and the cooperation of the health centers of Tehran. All participants were educated about the purpose of the study, and all procedures were conducted in accordance with the ethical standards of the Tehran University of Medical Sciences (No. IR.TUMS.VCR.REC.1397.157), which approved the protocol and informed consent form. All participants provided written informed consent prior to the start of the study.

\section{Dietary assessment}

Dietary intake was assessed using three 24-hour dietary recalls (24hDRs). All 24hDRs were collected by trained interviewers. The first recall was face-to-face during the participants' first visit at health centers. The other two $24 \mathrm{hDR}$ s were obtained on random days by phone interview. Portion sizes of consumed foods were converted to grams using household measures. ${ }^{17}$ Additionally, we used Nutritionist IV software (First Databank, San Bruno, CA, USA), modified for Iranian foods, to obtain the energy and nutrient values of food items.

\section{Breakfast definition}

Breakfast was defined as a meal eaten between 05:00 and 11:00. ${ }^{18}$

\section{Food group intake}

Dietitians independently classified foods and beverages into 24 groups of interest according to nutritional values, Iranian consumption habits, literary data, and previous experience of the research team. We then categorized food items into 14 food groups. 


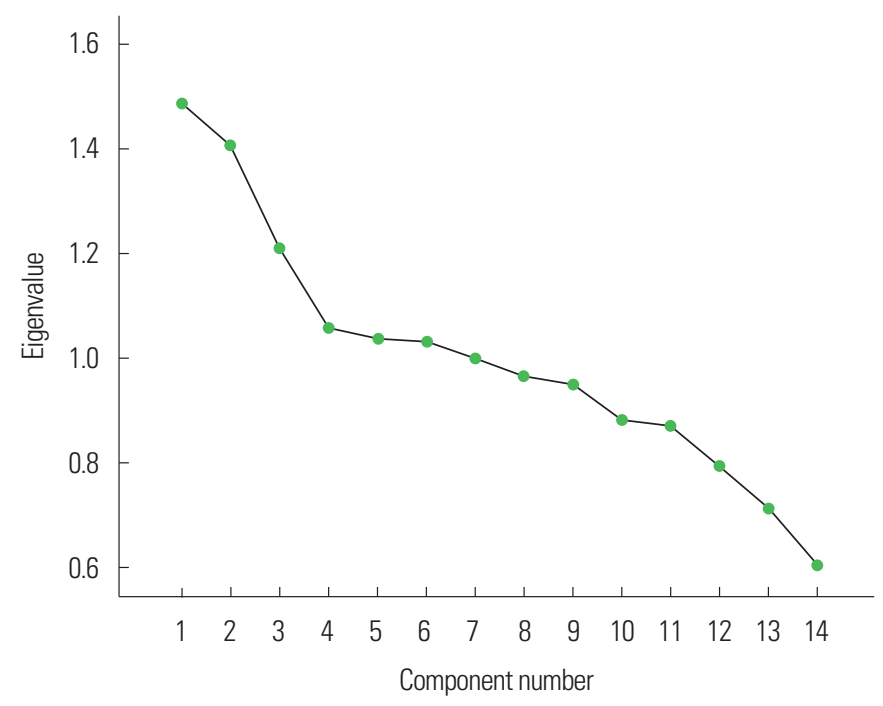

Figure 1. Scree plot for identification of dietary patterns by principal component analysis.

\section{Definition of brealkfast composition}

We obtained dietary patterns using principal component analysis (PCA; in particular, factor analysis) based on the usual intake for the 12 food groups. Food group intakes were energy-adjusted by the residual method. ${ }^{19}$ An orthogonal rotation method (Varimax rotation) was applied to simplify the factor structure and present it in an explainable manner. Food groups with a loading factor above an absolute value of 0.2 were considered to be important contributors to the dietary pattern. We retained three factors as shown in the scree plot shown in Fig. 1. We labeled dietary patterns based on food groups positively and negatively correlated with the identified patterns and based on prior literature. Then, scores for breakfast patterns were calculated as the sum of the products of the factor loading coefficients and intake of food groups weighted by their factor loadings. ${ }^{20}$ Each participant received a factor score for each identified pattern, and was categorized based on tertiles of major dietary pattern scores.

\section{Outcome assessment}

Weight and height were measured according to standardized methods. ${ }^{21}$ Weight was recorded to the nearest $100 \mathrm{~g}$, while minimally clothed and unshod, using digital scales. Height was measured in a standing position, unshod, using a tape stadiometer to the nearest $1 \mathrm{~mm}$ while the shoulders were in a normal position. Waist circumference (WC) was recorded to the nearest $0.1 \mathrm{~cm}$ at the um- bilical level, and hip circumference at the maximal level over light clothing, using a non-stretch tape measure without putting pressure on the body surface.

We assessed central obesity based on waist to hip ratio (WHR $\geq 0.9$ for men, $\geq 0.85$ for women) and WC (i.e., obesity if WC $\geq 102 \mathrm{~cm}$ for men, WC $\geq 88 \mathrm{~cm}$ for women). ${ }^{22} \mathrm{BMI}$ was calculated from height and weight data as $\mathrm{kg} / \mathrm{m}^{2}$. General obesity was defined as BMI $\geq 30 \mathrm{~kg} / \mathrm{m}^{2} .{ }^{23}$ Blood pressure was measured twice for each participant after a period of 15 minutes of quiet sitting. ${ }^{24}$

\section{Covariates}

Physical activity was assessed using the International short-form Physical Activity Questionnaire and from participants' oral responses to the international physical-activity questionnaire based on an interview, and subsequently expressed as metabolic equivalent $\mathrm{hr} / \mathrm{wk} .{ }^{25}$ We asked the participants to think about the vigorous and moderate activities that they had engaged in during the past 7 days and report the time they spent on these activities. Additional covariate information regarding age (year), education level (illiterate, under-diploma, diploma, educated), marital status (married or other), occupation (employee or unemployed), medical condition (healthy or underlying disease), smoking status (never smoker, former smoker, current smoker), and life-style (living alone, with someone) was obtained using questionnaires. Furthermore, we defined underlying diseases in this study as diabetes, hypertension, dyslipidemia, cardiovascular disease, cancer, and respiratory disease.

\section{Statistical analysis}

We used one-way analysis of variance for quantitative variables and chi-square tests for qualitative variables to determine the significance of differences across tertiles in breakfast patterns. To assess the association between breakfast composition and central and general obesity, respectively, we performed multiple logistic regression analyses. Analyses were adjusted for age (years), sex (male or female), life-style, marriage, physical activity level, smoking status (never smoke or former/current smoker), and total energy intake.. Data were analyzed using the statistical software package IBM SPSS version 22 (IBM Corp., Armonk, NY, USA) and statistical significance was accepted, a priori, at $P<0.05$. 
Table 1. Food groups used in the factor analysis and factor loadings for each of the identified breakfast patterns*

\begin{tabular}{|c|c|c|c|c|}
\hline \multirow[b]{2}{*}{ Food group } & \multirow[b]{2}{*}{ Food item } & \multicolumn{3}{|c|}{ Dietary pattern } \\
\hline & & $\begin{array}{l}\text { Bread and grains, } \\
\text { meat products, and coffee }\end{array}$ & $\begin{array}{l}\text { Sweets, tea } \\
\text { and coffee }\end{array}$ & $\begin{array}{l}\text { Fruits, vegetables, } \\
\text { and eggs }\end{array}$ \\
\hline Bread and grain & $\begin{array}{l}\text { White breads (lavash, baguettes), noodles, pasta, rice, toasted bread, white flour, } \\
\text { dark breads (e.g., barbari, sangak, taftun) }\end{array}$ & 0.701 & - & - \\
\hline Dairy product & $\begin{array}{l}\text { Low-fat milk, skim milk, low-fat yogurt, cheese, Kashk, yogurt drinks, high-fat milk, } \\
\text { high-fat yogurt, cream cheese, cream, dairy fat, ice cream, others }\end{array}$ & -0.387 & - & -0.397 \\
\hline Sweet & $\begin{array}{l}\text { Cookies, cakes, muffins, pies, chocolates, honey, jam, sugar cubes, sugar, candies, } \\
\text { sweet tahini, Iranian confectioneries (gaz, sohan, noghl), jam, jelly, others }\end{array}$ & - & 0.743 & - \\
\hline Egg & Eggs & - & -0.332 & 0.504 \\
\hline Fat & Hydrogenated fats, animal fats, butter, oils, olive oil, vegetable oils, olives & 0.643 & - & - \\
\hline Potato & Potatoes & - & - & 0.435 \\
\hline Processed meat & Sausage, hamburger, other & 0.443 & - & -0.209 \\
\hline Organ meat & Heart, kidney, liver, tongue, brain, & - & - & 0.204 \\
\hline Fruit and vegetable & $\begin{array}{l}\text { Melon, watermelon, honeydew melon, plums, prunes, apples, cherries, sour cherries, } \\
\text { peaches, nectarine, pear, fig, date, grapes, kiwi, pomegranate, strawberry, banana, } \\
\text { persimmon, berry, pineapple, oranges, dried fruits, all juices, cauliflower, carrot, } \\
\text { tomatoes and tomato products, spinach, lettuce, cucumber, eggplant, onion, greens, } \\
\text { green bean, green pea, squash, mushroom, pepper, corn, garlic, turnip, others }\end{array}$ & - & - & 0.564 \\
\hline Legume and nut & $\begin{array}{l}\text { Peanuts, almonds, pistachios, hazelnuts, roasted seeds, walnuts, lentils, split peas, } \\
\text { beans, chickpeas, fava beans, soy, others }\end{array}$ & - & -0.279 & -0.471 \\
\hline Coffee & Coffee & 0.215 & 0.730 & - \\
\hline Tea & Tea & -0.220 & 0.264 & - \\
\hline
\end{tabular}

*Factor loadings of $<0.2$ were removed to simplify the table.

\section{RESULTS}

In this study, of the 850 participants, 10 were excluded due to a lack of information and/or failure to complete their dietary recall report, thus 840 participants were included in the final analysis. The average daily total energy intake in the present study was 1,701.10; furthermore, $29 \%$ of the total energy intake was from breakfast. Factor loadings of food groups, the percentage of the distribution justified by each dietary pattern, and the identified dietary patterns are presented in Table 1 and Fig. 1. The first dietary pattern, named "bread and grains, meat products, and coffee," was rich in bread and grains, meat products (sausages and burgers), fats, and coffee. The second dietary pattern, "sweets, tea and coffee," included high consumption of sweets, desserts, tea and coffee. The third dietary pattern, "fruits, vegetables, and eggs" had higher amount of fruits and vegetables, potatoes, and eggs than the other two dietary patterns. These three dietary patterns explained $36.99 \%$ of the total variance of the studied population.

Correlation analysis revealed strong positive correlations between fat, bread, and grains $(P \leq 0.001)$ and between tea and sweets

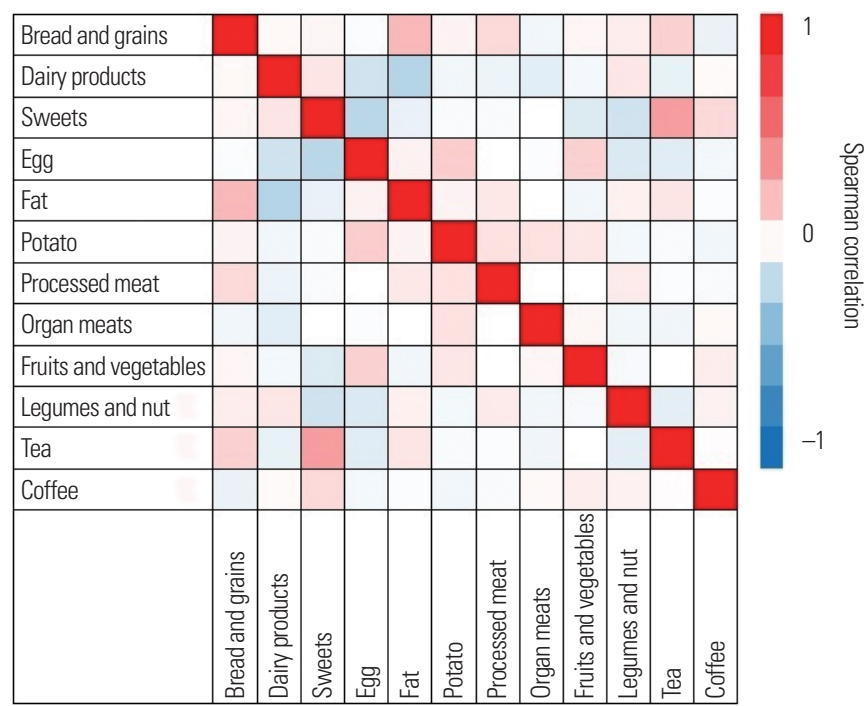

Figure 2. Heat map showing the Spearman correlation matrix for breakfast food intake in grams $(n=840)$ by food group. The color corresponds to the strength of correlations (red, positive correlation; white, no correlation; blue, negative correlation).

$(P \leq 0.001)$; in contrast, we found strong negative correlations between egg and sweet consumption $(P \leq 0.001)$ and between fat and dairy product consumption $(P \leq 0.001)$ (Fig. 2). 


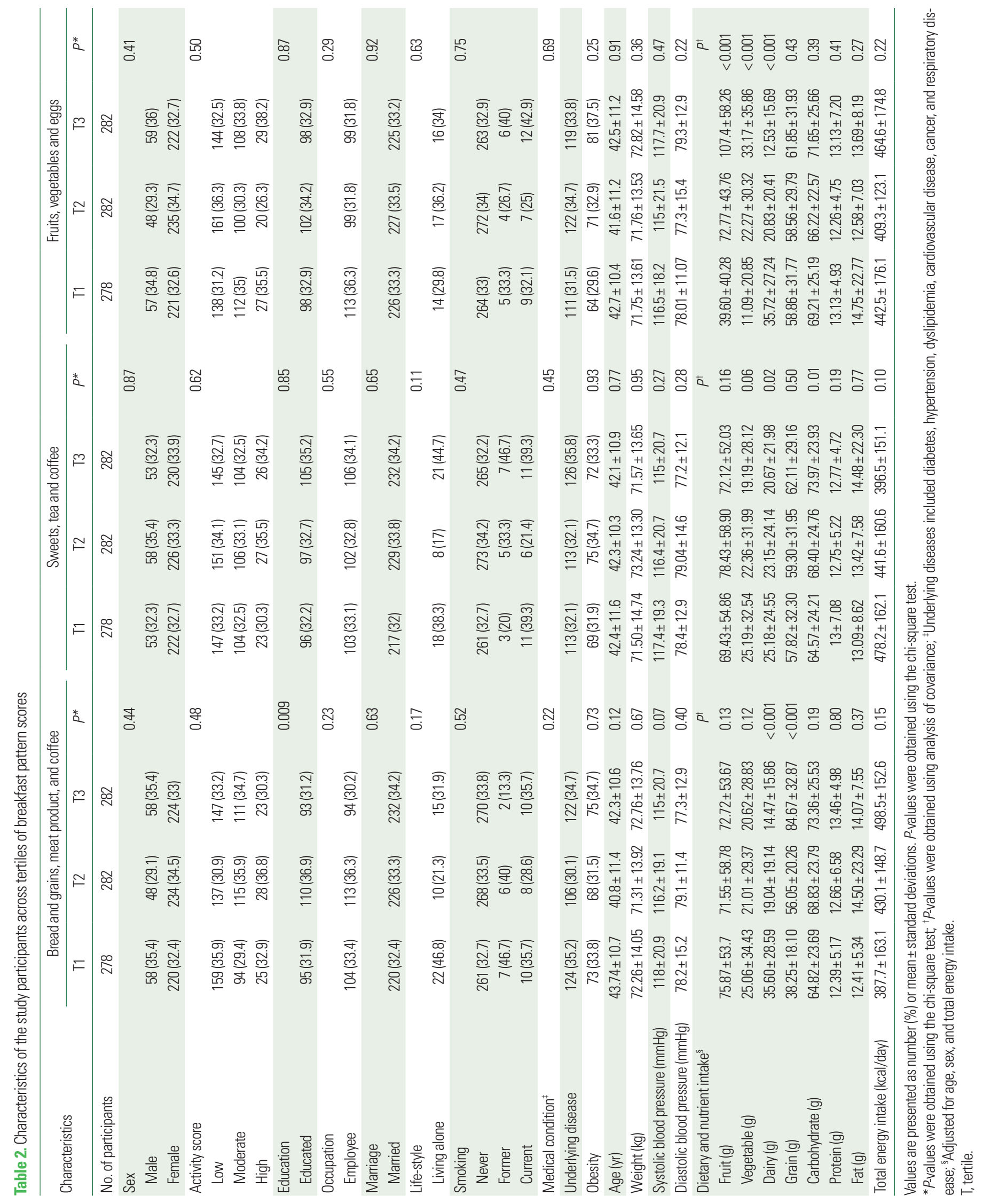


Table 2 shows the general characteristics, anthropometric characteristics, and dietary intake of participants for the three breakfast dietary patterns. Education level differed across tertiles of the dietary pattern "bread and grains, meat products, and coffee" $(P=$ 0.009); participants with higher education showed greater adherence to this dietary pattern. Analysis of covariance after controlling for age, sex, and total energy intake showed that mean intake of dairy products decreased while intake of grains increased with greater adherence to the "bread and grains, meat products, and coffee" dietary pattern. People in the highest tertile of the "sweets, tea and coffee" dietary pattern showed lower intake of dairy products and higher intake of carbohydrates. In addition, higher intake of fruit, vegetables, and lower intake of dairy products was observed in people with greater adherence to the "fruits, vegetables, and eggs" dietary pattern. There were no differences in the means of other variables across the three major dietary patterns.

Multivariable-adjusted means for anthropometric measures and indices across tertiles of breakfast patterns are presented in Supplementary Table 1 . Higher adherence to the dietary pattern of "bread and grains, meat products, and coffee" was associated with a higher WHR $(P=0.03)$. However, there was no significant relationship after controlling for confounding factors. The other dietary patterns did not show any significant relationships with any of these variables.
Unadjusted and adjusted odds ratios for general obesity among tertiles of dietary patterns are presented in Table 3 . There was no significant relationship between general obesity and any of the dietary patterns in the unadjusted model, and this remained true after adjusting for confounding factors.

Table 4 shows the unadjusted and adjusted odds ratios for central obesity among dietary pattern score tertiles. Adherence to the dietary pattern of "sweets, tea and coffee" yielded a 1.8-fold ( $P=$ $0.001)$ greater chance of a greater WC. However, the chance of central obesity in the two other dietary patterns showed no significant difference across tertiles of dietary patterns in the unadjusted and adjusted models. The chance of developing central obesity, defined as a WHR $\geq 0.90$ for men and $\geq 0.85$ for women showed that those who were in the highest tertile of the dietary pattern of "bread and grains, meat products, and coffee" compared to those in the first tertile had a lower chance of central obesity (WHR $\geq 0.90$ for men and $\geq 0.85$ for women; odds ratio, 0.66 ; $95 \%$ confidence interval, 0.47-0.92). However, after adjusting for confounding factors, the chance of central obesity increased by 1.67 -fold $(P=0.03)$ when defined based on WHR ( $\geq 0.90$ for men and $\geq 0.85$ for women). Finally, we found no significant relationship between adherence to any other dietary pattern and the chance of central obesity in the crude or adjusted models.

Table 3. Odds ratios and $95 \%$ confidence intervals for general obesity $\left(\mathrm{BMl} \geq 30 \mathrm{~kg} / \mathrm{m}^{2}\right)$ across tertiles of dietary pattern scores

\begin{tabular}{|c|c|c|c|c|c|c|}
\hline \multirow{2}{*}{ Variable } & \multicolumn{6}{|c|}{ Tertile of dietary pattern scores } \\
\hline & $\mathrm{T} 1(\mathrm{n}=278)$ & $\mathrm{T} 2(\mathrm{n}=282)$ & $P$ & $\mathrm{~T} 3(\mathrm{n}=282)$ & $P$ & Ptrend \\
\hline \multicolumn{7}{|c|}{ Bread and grains, meat product, and coffee } \\
\hline Model 1 & 1.00 (Reference) & $0.90(0.60-1.31)$ & 0.59 & $1.02(0.70-1.48)$ & 0.90 & 0.90 \\
\hline Model 2 & 1.00 (Reference) & $0.97(0.66-1.44)$ & 0.90 & $1.04(0.71-1.53)$ & 0.81 & 0.94 \\
\hline Model 3 & 1.00 (Reference) & $1.03(0.69-1.53)$ & 0.86 & $1.14(0.77-1.71)$ & 0.49 & 0.76 \\
\hline \multicolumn{7}{|c|}{ Sweets, tea and coffee } \\
\hline Model 1 & 1.00 (Reference) & $1.06(0.60-1.31)$ & 0.73 & $1.01(0.69-1.47)$ & 0.96 & 0.96 \\
\hline Model 2 & 1.00 (Reference) & $1.03(0.66-1.44)$ & 0.86 & $1.01(0.68-1.49)$ & 0.49 & 0.98 \\
\hline Model 3 & 1.00 (Reference) & $1.06(0.69-1.53)$ & 0.74 & $1.07(0.72-1.59)$ & 0.71 & 0.90 \\
\hline \multicolumn{7}{|c|}{ Fruits, vegetables and eggs } \\
\hline Model 1 & 1.00 (Reference) & $1.10(0.74-1.61)$ & 0.62 & $1.33(0.91-1.94)$ & 0.13 & 0.13 \\
\hline Model 2 & 1.00 (Reference) & $1.15(0.78-1.71)$ & 0.49 & $1.36(0.93-2.01)$ & 0.11 & 0.25 \\
\hline Model 3 & 1.00 (Reference) & $1.10(0.74-1.63)$ & 0.62 & $1.39(0.94-2.05)$ & 0.09 & 0.20 \\
\hline
\end{tabular}

Values are presented as odds ratio (95\% confidence interval). Odds ratios and confidence intervals were obtained using binary logistic regression. Model 1, unadjusted; Model 2, age, sex, education (categorical), marriage, life-style, smoking; Model 3, Model 2+physical activity and total energy intake.

BMI, body mass index; T, tertile. 
Table 4. Odds ratios and 95\% confidence intervals for central obesity across tertiles of dietary pattern scores

\begin{tabular}{|c|c|c|c|c|c|c|}
\hline \multirow{2}{*}{ Variable } & \multicolumn{6}{|c|}{ Tertile of dietary pattern scores } \\
\hline & $T 1(n=278)$ & $\mathrm{T} 2(\mathrm{n}=282)$ & $P$ & $\mathrm{~T} 3(\mathrm{n}=282)$ & $P$ & Ptrend \\
\hline \multicolumn{7}{|c|}{ Waist circumference $(\mathrm{cm})>102 \mathrm{~cm}$ for men and $>88 \mathrm{~cm}$ for women } \\
\hline \multicolumn{7}{|c|}{ Bread and grains, meat product, and coffee } \\
\hline Model 1 & 1.00 (Reference) & $0.80(0.57-1.11)$ & 0.19 & $0.90(0.65-1.26)$ & 0.55 & 0.55 \\
\hline Model 2 & 1.00 (Reference) & $0.86(0.60-1.23)$ & 0.42 & $0.93(0.65-1.33)$ & 0.71 & 0.74 \\
\hline Model 3 & 1.00 (Reference) & $0.87(0.61-1.25)$ & 0.47 & $0.96(0.67-1.40)$ & 0.86 & 0.78 \\
\hline \multicolumn{7}{|c|}{ Sweets, tea and coffee } \\
\hline Model 1 & 1.00 (Reference) & $1.22(0.87-1.71)$ & 0.23 & $1.66(1.19-2.32)$ & 0.003 & 0.23 \\
\hline Model 2 & 1.00 (Reference) & $1.26(0.88-1.81)$ & 0.19 & $1.78(1.24-2.55)$ & 0.002 & 0.006 \\
\hline Model 3 & 1.00 (Reference) & $1.30(0.90-1.88)$ & 0.15 & $1.80(1.61-2.59)$ & 0.001 & 0.005 \\
\hline \multicolumn{7}{|c|}{ Fruits, vegetables, and egg } \\
\hline Model 1 & 1.00 (Reference) & $0.86(0.61-1.20)$ & 0.37 & $1.04(0.75-1.45)$ & 0.80 & 0.80 \\
\hline Model 2 & 1.00 (Reference) & $0.87(0.61-1.24)$ & 0.46 & $1.10(0.77-1.57)$ & 0.59 & 0.40 \\
\hline Model 3 & 1.00 (Reference) & $0.86(0.60-1.23)$ & 0.42 & $1.11(0.77-1.58)$ & 0.56 & 0.35 \\
\hline \multicolumn{7}{|c|}{ Waist to hip ratio $>0.90$ for males and $>0.85$ for females } \\
\hline \multicolumn{7}{|c|}{ Bread and grains, meat product, and coffee } \\
\hline Model 1 & 1.00 (Reference) & $0.66(0.47-0.92)$ & 0.01 & $1.68(1.49-1.96)$ & 0.02 & 0.02 \\
\hline Model 2 & 1.00 (Reference) & $0.74(0.52-1.05)$ & 0.10 & $1.69(1.49-1.98)$ & 0.04 & 0.10 \\
\hline Model 3 & 1.00 (Reference) & $0.73(0.51-1.04)$ & 0.08 & $1.67(1.47-1.97)$ & 0.03 & 0.08 \\
\hline \multicolumn{7}{|c|}{ Sweets, tea and coffee } \\
\hline Model 1 & 1.00 (Reference) & $1.17(0.84-1.63)$ & 0.33 & $1.18(0.85-1.65)$ & 0.31 & 0.31 \\
\hline Model 2 & 1.00 (Reference) & $1.16(0.82-1.63)$ & 0.39 & $1.21(0.85-1.71)$ & 0.27 & 0.52 \\
\hline Model 3 & 1.00 (Reference) & $1.16(0.82-1.64)$ & 0.39 & $1.22(0.85-1.73)$ & 0.26 & 0.51 \\
\hline \multicolumn{7}{|c|}{ Fruits, vegetables, and eggs } \\
\hline Model 1 & 1.00 (Reference) & $0.85(0.61-1.18)$ & 0.33 & $1.02(0.73-1.43)$ & 0.86 & 0.86 \\
\hline Model 2 & 1.00 (Reference) & $0.91(0.65-1.29)$ & 0.62 & $1.04(0.74-1.47)$ & 0.79 & 0.73 \\
\hline Model 3 & 1.00 (Reference) & $0.92(0.65-1.30)$ & 0.65 & $1.05(0.74-1.48)$ & 0.77 & 0.75 \\
\hline
\end{tabular}

Values are presented as odds ratio (95\% confidence interval). Odds ratios and confidence intervals were obtained using binary logistic regression. Model 1, unadjusted; Model 2 , age, sex, education (categorical), marriage, life-style, smoking; Model 3, Model 2+physical activity and total energy intake.

T, tertile.

\section{DISCUSSION}

In the present study, we identified three major dietary patterns by factor analysis: the "bread and grains, meat products, and coffee" dietary pattern, the "sweets, tea and coffee" dietary pattern, and the "fruits, vegetables, and eggs" dietary pattern. According to our results, greater adherence to the dietary pattern of "sweets, tea and coffee" was associated with increased WC. In addition, a significant relationship was found between the dietary pattern of "bread and grains, meat products, and coffee" and WHR even though no significant relationship was observed between general obesity and breakfast dietary patterns.

Breakfast is traditionally considered not only the first meal, but also the most important meal of the day. ${ }^{26}$ Previous studies have re- ported that eating breakfast regularly leads to better diet quality, ${ }^{27}$ with reduced risk of obesity as well as cardio-metabolic diseases. ${ }^{28}$ van der Heijden et al..$^{29}$ showed that eating breakfast has a role in preventing weight gain while other studies have highlighted how not eating breakfast is associated with an increased risk of overweight and obesity, ${ }^{30}$ type 2 diabetes, ${ }^{31}$ and cardiovascular diseases. ${ }^{32}$ Therefore, eating a healthy breakfast has empirical, positive health consequences. ${ }^{33}$

In this study, we evaluated diet quality of breakfast in terms of dietary patterns. Knowledge of dietary patterns, which take into consideration different aspects of dietary behaviors, can provide more accurate data for evaluating the relationship between diet and health state compared to the study of nutrients or foods in isolation. ${ }^{34}$ Identifying dietary patterns is also considered the best approach to 
determine the effects of particular nutrients and specific foods on health. ${ }^{7,35}$ Such an approach allows for the synergistic effects of nutrients and foods to be considered, ${ }^{36}$ particularly when combined with PCA for statistical discernment and evaluation of dietary patterns. ${ }^{37}$

In the present study, three dominant dietary patterns were identified in the breakfast meals of Tehranese adults. The first dietary pattern was "bread and grains, meat products, and coffee", characterized by high consumption of bread and grains, meat products (sausages and burgers), fats, and coffee. The second dietary pattern was termed, "sweets, tea and coffee," and included high consumption of sweets, desserts, tea, and coffee. The third dietary pattern was called "fruits, vegetables, and eggs", and contained high intake of fruits, vegetables, potatoes, and eggs. Baltar et al..$^{38}$, reported a southeastern Brazilian dietary pattern consisting of cold-cut meats, milk, cheese, coffee/tea, bread) similar to our "bread and grains, meat products, and coffee" dietary pattern. The snack diet pattern (positive factor load for cold cuts, yellow cheese and bread/toast, and an inverse association with salted biscuits) reported by de Oliveira Santos et al..$^{39}$ is similar to the "bread and grains, meat products, and coffee" dietary pattern.

In addition, the dietary pattern of "sweets, tea and coffee" in our study is similar to the traditional dietary pattern reported by Chatelan et al. ${ }^{9}$ of white bread, butter, and sweet spread, and the third dietary pattern identified by Iqbal et al. ${ }^{40}$ (high factor load for sugar and confectionary, eggs, butter, and bread). Furthermore, the dietary pattern of "fruits, vegetables, and eggs" in our study is similar to the second dietary pattern ("prudent"-fruit, unprocessed and unsweetened cereal flakes, nuts/seeds, yogurt) in the study of Chatelan et al. ${ }^{9}$ as well as the Northern Brazil dietary pattern reported by Baltar et al. ${ }^{38}$ (positive factor loadings for meats, preparations with corn, eggs, tubers/roots/potatoes, dairy products, savory snacks/crackers, fruit juices/fruit drinks/soy-based drinks).

However, the identified components of dairy products and snacks for the "fruits, vegetables, and eggs" dietary pattern differed between our study and that of Baltar et al..$^{38}$ One of the possible reasons for this difference is the strong dependence of the results on the population being studied. There are likely to be significant differences in dietary patterns according to geographical region, race, and culture. However, factor analysis also has some limitations due to the theo- retical or optional decisions that the researcher makes regarding the classification of foods, the number of factors that need to be preserved, and their naming; these decisions can also partially influence findings. Therefore, it is necessary to evaluate dietary patterns of people within individual countries and determine their relationship with health-disease status within these individual countries.

According to our results, those who consumed "bread and grains, meat products, and coffee" or "sweets, desserts, tea and coffee" for the breakfast meal had a higher risk of increased WC and WHR. These patterns can be considered unhealthy dietary patterns. Some studies have shown results consistent with the present study; for instance, in the study by Rezazadeh and Rashidkhani ${ }^{41}$ on women in northeastern Tehran and the study by Esmaillzadeh and Azadbakht ${ }^{42}$ on Tehranese female teachers, it was reported that unhealthy dietary patterns increased the risk of general and central obesity, whilst healthy dietary patterns reduced the risk. ${ }^{42}$ In contrast, a study by Keramati et al. ${ }^{43}$ reported higher adherence to a dietary pattern that was characterized by high consumption of fried potatoes, mayonnaise, sweets, desserts, and liquid oils was associated with s lower BMI. In addition, the southeastern Brazilian dietary pattern identified by Baltar et al., ${ }^{38}$ which is comparable to the dietary pattern of "bread and grains, meat products, and coffee" in the present study, showed an inverse relationship with obesity, inconsistent with our study results. The possible reason for the inverse relationship of the Brazilian southeast dietary pattern with obesity among Brazilians may be due to the combination of this dietary pattern with lower levels of fat and sugar, and good sources of protein, calcium, and nutrients associated with lower weight gain. The positive relationship between the dietary pattern of "bread and grains, meat products, and coffee" in the present study and obesity may be due to the high fat content in this pattern.

The positive relationship between unhealthy dietary patterns with central obesity in the present study is likely due to high consumption of simple sugars (sugar, sweets and desserts) and high fat foods (butter, cream, cream cheese, solid oil, liquid oil, olive oil, olive, animal fat, animal oil, and margarine), which are high energy but low nutrient density foods. Eating high fat foods, which is another feature of an unhealthy eating pattern, can lead to overweight and obesity by exacerbating inactivity and increasing dietary energy density. ${ }^{44}$ The healthy dietary pattern in the present study was not 
significantly correlated with obesity and central obesity. Some other studies, such as those of Rezazadeh and Rashidkhani ${ }^{41}$ and Shin et al. ${ }^{45}$ showed that healthy dietary patterns were inversely related to the risk of overweight or obesity. However, the "prudent" dietary pattern identified in the study of Chatelan et al., ${ }^{9}$ which is similar to the dietary pattern of "fruits, vegetables, and eggs" in our study, had an inverse relationship with central obesity, inconsistent with our findings. This may be due to different factor loadings in the two studies. For example, in the present study, the factor loadings of legumes and nuts were negative, but in Chatelan et al., ${ }^{9}$ the factor loadings of nuts and seeds were positive.

There is an expected inverse relationship between a healthy diet and the risk of obesity. However, the "fruits, vegetables, and eggs" dietary pattern in the present study includes some factor loadings containing fiber and high glycemic index foods. According to the available evidence, fiber is an important factor in this pattern. Medium and high fiber foods, such as cooked cereals, fruits, and vegetables remain longer in the gastrointestinal tract than low fiber foods, leading to prolonged satiety. ${ }^{46}$ Therefore, eating high fiber foods may prevent or reduce the consumption of high fat foods. ${ }^{47}$ In addition, high glycemic index foods, such as potatoes, are associated with obesity, and high glycemic index foods increase blood glucose levels, subsequently leading to high insulin secretion characterized by the rapid release of insulin and blood sugar reduction, resulting in hunger, overeating, and obesity. ${ }^{48}$

One of the strengths of the present study is that it is the first study conducted in Iran to examine the effect of dietary meal patterns and obesity. Moreover, our sample number was higher than previous studies that examined dietary patterns in Iran. However, a limitation of this study is the cross-sectional nature of the study, which precludes determination of a causal relationship. This highlights the need for further well-controlled investigations. Overall, adherence to specific dietary patterns for the breakfast meal may increase the chance of central obesity in Iranian adults. Thus, careful consideration should be given to the composition of breakfast at a population level.

\section{CONFLICTS OF INTEREST}

The authors declare no conflict of interest.

\section{ACKNOWLEDGMENTS}

This research was supported by a grant from Tehran University of Medical Sciences (No. 40186).

Authors thank all those who participated in this study.

\section{AUTHOR CONTRIBUTIONS}

Study concept and design: SSB; acquisition of data: ZA; analysis and interpretation of data: ZA, PG and MM; drafting of the manuscript: ZA and SM; critical revision of the manuscript: $\mathrm{KD}, \mathrm{SSB}$, and CCTC; statistical analysis: SSB, ZA; obtained funding: SSB; administrative, technical, or material support: SSB; and study supervision: all authors.

\section{SUPPLEMENTARY MATERIALS}

Supplementary Table 1 can be found via https://doi.org/10. $7570 /$ jomes 20042.

\section{REFERENCES}

1. Ayatollahi SM, Ghoreshizadeh Z. Prevalence of obesity and overweight among adults in Iran. Obes Rev 2010;11:335-7.

2. Alwan A. Global status report on noncommunicable diseases 2010. Geneva: World Health Organization; 2011.

3. Karpe F, Pinnick KE. Biology of upper-body and lower-body adipose tissue: link to whole-body phenotypes. Nat Rev Endocrinol 2015;11:90-100.

4. Flegal KM, Kit BK, Orpana H, Graubard BI. Association of all-cause mortality with overweight and obesity using standard body mass index categories: a systematic review and metaanalysis. JAMA 2013;309:71-82.

5. Bastien M, Poirier P, Lemieux I, Després JP. Overview of epidemiology and contribution of obesity to cardiovascular disease. Prog Cardiovasc Dis 2014;56:369-81.

6. Cameron AJ, Magliano DJ, Shaw JE, Zimmet PZ, Carstensen B, Alberti KG, et al. The influence of hip circumference on the relationship between abdominal obesity and mortality. Int J Epidemiol 2012;41:484-94. 
7. Hu FB. Dietary pattern analysis: a new direction in nutritional epidemiology. Curr Opin Lipidol 2002;13:3-9.

8. Hopkins LC, Sattler M, Steeves EA, Jones-Smith JC, Gittelsohn J. Breakfast consumption frequency and its relationships to overall diet quality, using healthy eating index 2010, and body mass index among adolescents in a low-income urban setting. Ecol Food Nutr 2017;56:297-311.

9. Chatelan A, Castetbon K, Pasquier J, Allemann C, Zuber A, Camenzind-Frey E, et al. Association between breakfast composition and abdominal obesity in the Swiss adult population eating breakfast regularly. Int J Behav Nutr Phys Act 2018;15: 115.

10. Navia B, López-Sobaler AM, Villalobos T, Aranceta-Bartrina J, Gil Á, González-Gross M, et al. Breakfast habits and differences regarding abdominal obesity in a cross-sectional study in Spanish adults: The ANIBES study. PLoS One 2017;12:e0188828.

11. Mansouri M, Hasani-Ranjbar S, Yaghubi H, Rahmani J, Tabrizi YM, Keshtkar A, et al. Breakfast consumption pattern and its association with overweight and obesity among university students: a population-based study. Eat Weight Disord 2020;25: 379-87.

12. Sa J, Heimdal J, Sbrocco T, Seo DC, Nelson B. Overweight and physical inactivity among African American students at a historically black university. J Natl Med Assoc 2016;108:77-85.

13. Astbury NM, Taylor MA, Macdonald IA. Breakfast consumption affects appetite, energy intake, and the metabolic and endocrine responses to foods consumed later in the day in male habitual breakfast eaters. J Nutr 2011;141:1381-9.

14. Kobayashi F, Ogata H, Omi N, Nagasaka S, Yamaguchi S, Hibi M, et al. Effect of breakfast skipping on diurnal variation of energy metabolism and blood glucose. Obes Res Clin Pract 2014;8:e201-98.

15. Sievert K, Hussain SM, Page MJ, Wang Y, Hughes HJ, Malek M, et al. Effect of breakfast on weight and energy intake: systematic review and meta-analysis of randomised controlled trials. BMJ 2019;364:142.

16. Min C, Noh H, Kang YS, Sim HJ, Baik HW, Song WO, et al. Breakfast patterns are associated with metabolic syndrome in Korean adults. Nutr Res Pract 2012;6:61-7.

17. Ghaffarpour M, Houshiar-Rad A, Kianfar H. The manual for household measures, cooking yields factors and edible portion of food. Tehran: Keshaverzi Press; 1999.

18. Kahleova H, Lloren JI, Mashchak A, Hill M, Fraser GE. Meal frequency and timing are associated with changes in body mass index in adventist health study 2. J Nutr 2017;147:1722-8.

19. Willett W. Nutritional Epidemiology. New York (NY): Oxford University Press; 1998.

20. Kim JO, Mueller CW. Factor analysis: Statistical methods and practical issues. Beverly Hills (CA): Sage; 1978.

21. Lohman TG, Roche AF, Martorell R. Anthropometric standardization reference manual. Champaign (IL): Human kinetics books; 1988.

22. World Health Organization. Waist circumference and waist-hip ratio: report of a WHO expert consultation. Geneva: World Health Organization; 2011.

23. Amini M, Shafaeizadeh S, Zare M, Khosravi Boroujeni H, Esmaillzadeh A. A cross-sectional study on food patterns and adiposity among individuals with abnormal glucose homeostasis. Arch Iran Med 2012;15:131-5.

24. Azizi F, Emami H, Salehi P, Ghanbarian A, Mirmiran P, Mirbolooki $\mathrm{M}$, et al. Cardiovascular risk factors in the elderly: the Tehran Lipid and Glucose Study. J Cardiovasc Risk 2003; 10:65-73.

25. Ainsworth BE, Haskell WL, Whitt MC, Irwin ML, Swartz AM, Strath SJ, et al. Compendium of physical activities: an update of activity codes and MET intensities. Med Sci Sports Exerc 2000;32(9 Suppl):S498-504.

26. Siega-Riz AM, Popkin BM, Carson T. Differences in food patterns at breakfast by sociodemographic characteristics among a nationally representative sample of adults in the United States. Prev Med 2000;30:415-24.

27. Goon S, Islam S. Breakfast skipping and obesity risk among urban adults in Bangladesh. J Int J Public Health Sci 2014;3: $15-22$

28. Keski-Rahkonen A, Kaprio J, Rissanen A, Virkkunen M, Rose RJ. Breakfast skipping and health-compromising behaviors in adolescents and adults. Eur J Clin Nutr 2003;57:842-53.

29. van der Heijden AA, Hu FB, Rimm EB, van Dam RM. A prospective study of breakfast consumption and weight gain among U.S. men. Obesity (Silver Spring) 2007;15:2463-9. 
30. Bazzano LA, Song Y, Bubes V, Good CK, Manson JE, Liu S. Dietary intake of whole and refined grain breakfast cereals and weight gain in men. Obes Res 2005;13:1952-60.

31. Mekary RA, Giovannucci E, Willett WC, van Dam RM, Hu FB. Eating patterns and type 2 diabetes risk in men: breakfast omission, eating frequency, and snacking. Am J Clin Nutr 2012; 95:1182-9.

32. Cahill L, Chiuve S, Mekary R, Jensen M, Flint A, Hu F, et al. Breakfast eating and incident coronary heart disease in a large prospective cohort of American women. FASEB J 2015;29: 906-3.

33. Adamsson V, Reumark A, Marklund M, Larsson A, Risérus U. Role of a prudent breakfast in improving cardiometabolic risk factors in subjects with hypercholesterolemia: a randomized controlled trial. Clin Nutr 2015;34:20-6.

34. Bach-Faig A, Geleva D, Carrasco J, Ribas-Barba L, Serra-Majem L. Evaluating associations between Mediterranean diet adherence indexes and biomarkers of diet and disease. Public Health Nutr 2006;9:1110-7.

35. Ocké MC. Evaluation of methodologies for assessing the overall diet: dietary quality scores and dietary pattern analysis. Proc Nutr Soc 2013;72:191-9.

36. Andersson J, Nydahl M, Gustafsson K, Sidenvall B, Fjellström C. Meals and snacks among elderly self-managing and disabled women. Appetite 2003;41:149-60.

37. Beck KL, Jones B, Ullah I, McNaughton SA, Haslett SJ, Stonehouse W. Associations between dietary patterns, socio-demographic factors and anthropometric measurements in adult New Zealanders: an analysis of data from the 2008/09 New Zealand Adult Nutrition Survey. Eur J Nutr 2018;57:1421-33. 38. Baltar VT, Cunha DB, Santos RO, Marchioni DM, Sichieri R. Breakfast patterns and their association with body mass index in Brazilian adults. Cad Saude Publica 2018;34:e00111917.
39. de Oliveira Santos R, Fisberg RM, Marchioni DM, Troncoso Baltar V. Dietary patterns for meals of Brazilian adults. Br J Nutr 2015;114:822-8.

40. Iqbal K, Schwingshackl L, Gottschald M, Knüppel S, StelmachMardas M, Aleksandrova K, et al. Breakfast quality and cardiometabolic risk profiles in an upper middle-aged German population. Eur J Clin Nutr 2017;71:1312-20.

41. Rezazadeh A, Rashidkhani B. The association of general and central obesity with major dietary patterns of adult women living in Tehran, Iran. J Nutr Sci Vitaminol (Tokyo) 2010;56: 132-8.

42. Esmaillzadeh A, Azadbakht L. Major dietary patterns in relation to general obesity and central adiposity among Iranian women. J Nutr 2008;138:358-63.

43. Keramati M, Shariati Bafghi E, Rajaii AR, Rashidkhani B. The association between dietary patterns and bone mineral density in a sample of postmenopausal women living in Tehran. J Nutr Sci Food Technol 2012;7:51-62.

44. Bray GA, Popkin BM. Dietary fat intake does affect obesity! Am J Clin Nutr 1998;68:1157-73.

45. Shin KO, Oh SY, Park HS. Empirically derived major dietary patterns and their associations with overweight in Korean preschool children. Br J Nutr 2007;98:416-21.

46. Turconi G, Bazzano R, Caramella R, Porrini M, Crovetti R, Lanzola E. The effects of high intakes of fibre ingested at breakfast on satiety. Eur J Clin Nutr 1995;49 Suppl 3:S281-5.

47. Cho S, Dietrich M, Brown CJ, Clark CA, Block G. The effect of breakfast type on total daily energy intake and body mass index: results from the Third National Health and Nutrition Examination Survey (NHANES III). J Am Coll Nutr 2003; 22:296-302.

48. Ludwig DS. Dietary glycemic index and obesity. J Nutr 2000; 130(2S Suppl):280S-283S. 